\title{
CADASIL presenting as a change in personality
}

\author{
Champika Gamakaranage ${ }^{1}$, Thashi Chang ${ }^{2}$ \\ Journal of the Ceylon College of Physicians, 2011, 42, 33-34
}

\section{Introduction}

Cerebral autosomal dominant arteriopathy with subcortical infarcts and leukoencephalopathy (CADASIL) is a rare, autosomal dominant angiopathy, that is characterised by recurrent strokes and progressive vascular cognitive impairment. It is caused by mutations in the NOTCH3 gene on chromosome $19^{1}$. The prevalence of mutation carriers has been estimated at 1 in 50,000 to 1 in 121,000 individuals ${ }^{2}$. Patients with CADASIL usually present with ischaemic cerebral events, cognitive deficits, migraine with aura and psychiatric disturbances. However, varying phenotypic expression often leads to under recognition. We report a case of CADASIL presenting as a change in personality.

\section{Case report}

A 55-year old man with a past history of diabetes mellitus and dyslipidaemia for one year sought medical attention because of non-specific, transient episodes of dizziness. During the interview, his wife volunteered that there was recent change in personality, with mood swings, aggressive behaviour and talking to self. There were no features to suggest a diagnosis of mania, depression or psychosis. Further questioning revealed a strong family history of early onset strokes, psychiatric disease, dementia and premature deaths from these conditions among siblings, cousins and his mother's siblings. A total of six family members within two generations were affected (Figure 1). There was no past history of optic neuritis or transient neurological deficits.

He scored 19/30 on the Montreal Cognitive Assessment (MoCA) test (normal >26/30) although his minimental state examination score $(28 / 30)$ was normal. The neurological examination was unremarkable. Systemic examination was normal. His blood pressure was 120/80 mmHg.

\footnotetext{
${ }^{1}$ Registrar in Medicine,

${ }^{2}$ Neurologist, University Medical Unit, National Hospital of Sri Lanka, Colombo.
}

Routine haematological and biochemical tests including VDRL, HIV antibody and thyroid function tests were normal. The MRI of the brain showed multifocal, subcortical white matter lesions, most prominent in the periventricular region, suggestive of differential diagnoses of multiple sclerosis, CADASIL or multifocal small vessel disease.

\section{Discussion}

CADASIL was diagnosed in our patient based on the clinical presentation of behavioural change, cognitive deficit detected on the MoCA test and the association of a strong family history of early onset strokes, dementia and psychiatric manifestations, and the typical findings on MRI.

The usual clinical progression in CADASIL tends to occur with the sequential development of migraine with aura around age 30, TIAs, ischemic strokes and mood disorders between 40 and 60 years and dementia between 50 and 60 years, albeit being highly variable even within families. An early onset of disease does not necessarily predict rapid progression ${ }^{3}$.

The onset of MRI-visible lesions and the rate of lesion progression are variable, but by age 35 years all mutation carriers have developed MRI lesions ${ }^{4}$. Small irregular T2-hyperintensities involving the periventricular and deep white matter are usually the first sign. Temporal pole white matter hyperintensities seen on T2-weighted sequences (Figure 2), as seen in our patient, are found in about 90 percent of patients with CADASIL, whereas such lesions are uncommon in sporadic small vessel disease ${ }^{5}$.

The diagnosis is confirmed by genetic analysis for NOTCH3 mutation or by identifying granular osmiophilic material within the vascular basal lamina in skin biopsy on electron microscopy. These tests were not done in our patient because of unavailability. However, six months follow up of our patient since presentation has not suggested an alternate diagnosis.

The protein product NOTCH3 is critical for vascular smooth muscle cell differentiation and vascular development. The underlying vascular lesion in 


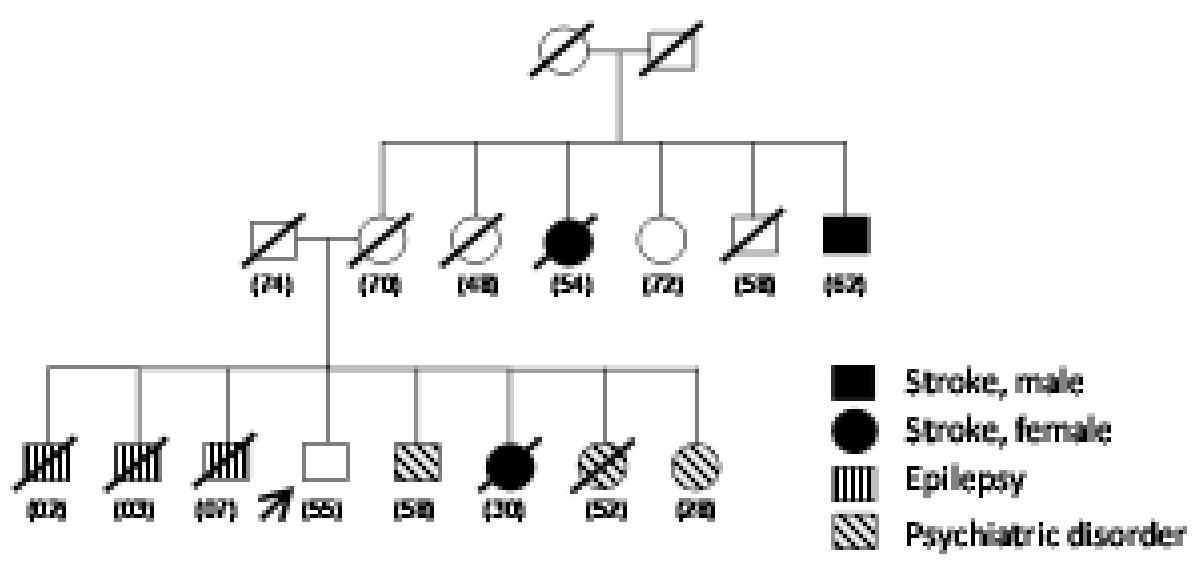

Figure 1. The patient's pedigree chart showing a strong family history of strokes, psychiatric disorders and epilepsy among his siblings and maternal siblings. Age in years is shown within parentheses.
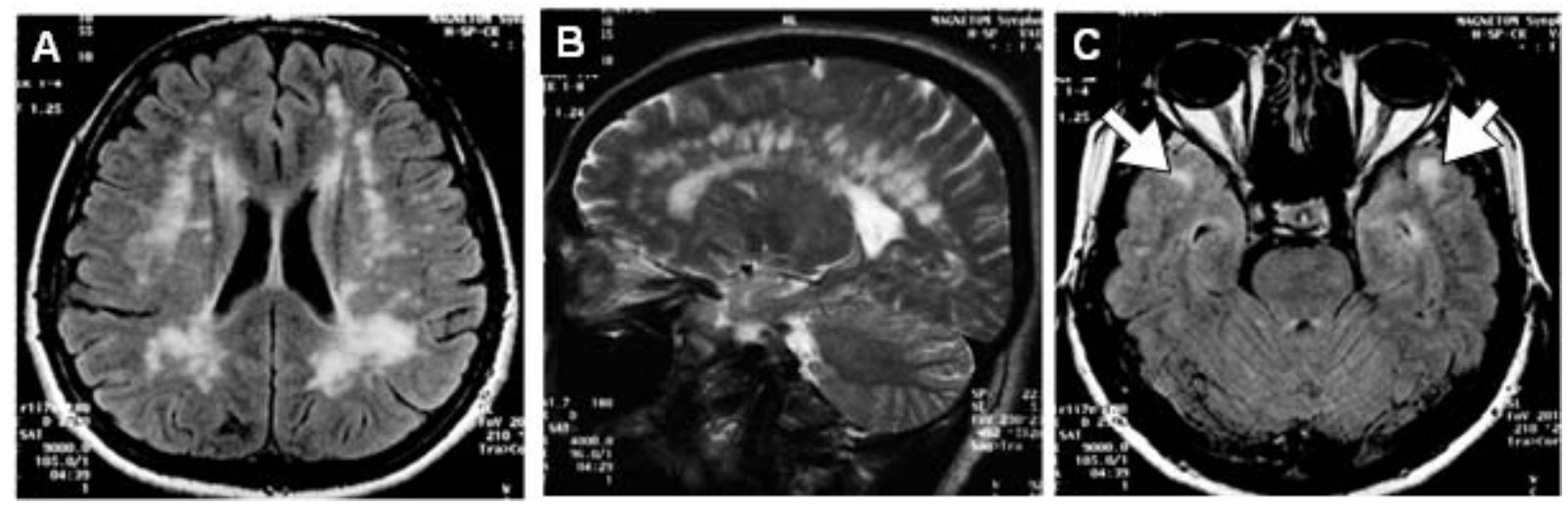

Figure 2. MRI of the brain with fluid-attenuated inversion-recovery (FLAIR) show high-signal intensity lesions (A) in the periventricular, subcortical white matter and (B) mimicking Dawson's fingers in the sagittal view reminiscent of multiple sclerosis. However, (C) high-signal intensity lesions in the temporal poles (arrows) are characteristic of CADASIL.

CADASIL is a unique non-arteriosclerotic, amyloidnegative angiopathy involving small arteries and capillaries. Although CADASIL is a generalized angiopathy, the vascular complications are largely limited to the brain.

Our case adds to the wide-spectrum of clinical presentation of CADASIL and highlights the need for a high index of suspicion in recognizing the disorder.

\section{References}

1. Joutel A, Corpechot C, Ducros A, et al. Notch3 mutations in CADASIL, a hereditary adult-onset condition causing stroke and dementia. Nature 1996; 383: 707.
2. Razvi SS, Davidson R, Bone I, Muir KW. The prevalence of cerebral autosomal dominant arteriopathy with subcortical infarcts and leucoencephalopathy (CADASIL) in the west of Scotland. J Neurol Neurosurg Psychiatry 2005; 76: 739.

3. Dichgans M, Mayer M, Uttner I, et al. The phenotypic spectrum of CADASIL: clinical findings in 102 cases. Ann Neurol 1998; 44: 731.

4. Chabriat $\mathrm{H}$, Vahedi K, Iba-Zizen MT, et al. Clinical spectrum of CADASIL: a study of 7 families. Cerebral autosomal dominant arteriopathy with subcortical infarcts and leukoencephalopathy. Lancet 1995; 346: 934.

5. O'Sullivan M, Jarosz JM, Martin RJ, et al. MRI hyperintensities of the temporal lobe and external capsule in patients with CADASIL. Neurology 2001; 56: 628. 